\title{
Malignancy history affected the prognosis of COVID-19 patients via release of Interleukin-6
}

\author{
Xiaowu Shi ${ }^{1}$, Jiahao $\mathrm{Hu}^{1}$, Haixia Ding ${ }^{2}$, Shenglan $\mathrm{Ye}^{1}$, Guoxing $\mathrm{Xu}^{1}$, Xiuwen Yang ${ }^{1}$, and \\ Liangchao Wang ${ }^{1}$ \\ ${ }^{1}$ Huazhong University of Science and Technology Tongji Medical College \\ ${ }^{2}$ Wuhan University Zhongnan Hospital
}

July 6,2020

\begin{abstract}
Background: Coronavirus disease 2019 (COVID-19), a newly erupted respiratory infectious disease caused by severe acute respiratory syndrome coronavirus 2 (SARS-CoV-2), has swept across the most of countries. The laboratory characteristics of COVID-patients accompanied with cancer and the risk factors for disease progression and survival of this particular population were few reported. Methods: We enrolled 585 confirmed COVID-19 patients admitted to our hospitals with measured interleukin-6 level on admission. Laboratory tests and outcome were extracted from electronic medical records. Data was divided to cancer group and non-cancer group to explorer the risk factors of progression and survival. Findings: A total of 44 patients with different cancer type (cancer group) and 487 patients without cancer (non-cancer group) were included. Cancer group had significant higher levels of NEUT, NLR, IL-6, and CRP than non-cancer group, but lymphocyte count and ALB were lower. Cancer group showed significantly higher progression rate $(42 \cdot 1 \%$ vs $22 \cdot 5 \%)$ and mortality $(27 \cdot 27 \%$ vs $11.91 \%)$ than non-cancer group. Elevated IL-6 and CRP were the risk factors associated with progression among moderate patients and death in-hospital (all $\mathrm{p}<0.05$ ) in non- cancer group. This correlation was not observed in caner group. Interpretation: IL-6, CRP, NEUT, and NLR were elevated in COVID-19 patients with cancer, with lower level of LYMP and ALB. IL-6 and CRP were positively correlated with progression and poor outcome in patients without cancer. As one of combined diseases, despite malignancy history did not directly affect the prognosis of COVID-19, but it could play a role in the poorer outcome through release of IL-6 and CRP.
\end{abstract}

\section{Hosted file}

IL6 Main Document.pdf available at https://authorea.com/users/340277/articles/467359malignancy-history-affected-the-prognosis-of-covid-19-patients-via-release-ofinterleukin-6 
Table 1: Demographic, laboratory findings, and outcomes of COVID-19 patients.

\begin{tabular}{|c|c|c|c|}
\hline & $\begin{array}{l}\text { Cancer } \\
(\mathrm{n}=44)\end{array}$ & $\begin{array}{l}\text { Non-cancer } \\
(\mathrm{n}=541)\end{array}$ & $P$ value \\
\hline Age, years & 67 (IQR: 54-80.5) & 59 (IQR: 42-69) & 0.005 \\
\hline \multicolumn{4}{|l|}{ Gender } \\
\hline Female (\%) & $20(45.5)$ & $294(54.3)$ & \multirow{2}{*}{0.255} \\
\hline Male (\%) & $24(54.5)$ & $247(45.7)$ & \\
\hline Time in-hospital, days & 31 (IQR: 19-48.5) & 21 (IQR: 13-31) & $<0.001$ \\
\hline \multicolumn{4}{|l|}{ Laboratory findings } \\
\hline $\mathrm{WBC}, \times 10^{9} / \mathrm{L}$ & 6.15 (IQR: $4.55-7.51)$ & 5.37 (IQR: $4.30-6.87$ ) & 0.065 \\
\hline NEUT, $\times 10^{9} / \mathrm{L}$ & 4.18 (IQR: $2.89-6.32)$ & 3.43 (IQR: 2.59-4.80) & 0.015 \\
\hline LYMP,$\times 10^{9} / \mathrm{L}$ & 0.85 (IQR: $0.58-1.40)$ & 1.26 IQR: $(0.84-1.71)$ & 0.001 \\
\hline NLR & 5.15 (IQR: $2.53-8.52)$ & 2.61 (IQR: 1.68-4.92) & 0.001 \\
\hline ALB, $g / L$ & 36.8 (IQR: $31.7-42.2$ ) & 39.5 (IQR: 35.15-43.2) & 0.017 \\
\hline IL-6, pg/L & 13.9 (IQR: 6.24-32.4) & 4.19 (IQR: 2.15-12.23) & $<0.001$ \\
\hline $\mathrm{CRP}, \mathrm{mg} / \mathrm{dl}$ & 2.96 (IQR: $0.38-6.32)$ & 0.59 (IQR: 0.11-3.37) & $<0.001$ \\
\hline \multicolumn{4}{|l|}{ Severity } \\
\hline Moderate (\%) & $38(86.4)$ & $472(87.2)$ & \multirow{2}{*}{0.866} \\
\hline Severe/Critical (\%) & $6(13.6)$ & $69(12.8)$ & \\
\hline \multicolumn{4}{|c|}{ Progression among moderate patients } \\
\hline Stabilization (\%) & $22(57.9)$ & $366(77.5)$ & \multirow{2}{*}{0.006} \\
\hline Poor progression (\%) & $16(42.1)$ & $106(22.5)$ & \\
\hline \multicolumn{4}{|l|}{ Outcomes } \\
\hline Survivor (\%) & $32(72.73)$ & $429(88.09)$ & \multirow{2}{*}{0.001} \\
\hline Non-survivor (\%) & $12(27.27)$ & $58(11.91)$ & \\
\hline
\end{tabular}

Note: Quantitative vales coincided with normal distribution are expressed by mean $\pm \mathrm{SD}$, and median (interquartile range, IQR) for the non-normal distribution data. Frequency (percentage) was used to express the counting data.

$P$-values: result from Chi-square test (for gender, severity, progression among moderate patients and outcomes) and Mann-Whitney U-test (for age, time in-hospital and laboratory findings).

Abbreviations: WBC, white blood cell count; NEUT, neutrophil count; LYMP, lymphocyte count; NLR,

neutrophil-to-lymphocyte ratio; ALB, serum albumin; IL-6, interleukin-6; CRP, C-reactive protein. 